\title{
Age-related changes in spatial navigation are evident by midlife and differ by sex
}

\author{
Shuying $\mathrm{Yu}^{1}$, Alexander P. Boone ${ }^{1+}$, Chuanxiuyue $\mathrm{He}^{1}$, Rie C. Davis ${ }^{4}$, Mary Hegarty ${ }^{1 *}$, \\ Elizabeth R. Chrastil ${ }^{2,4 *}$, Emily G. Jacobs ${ }^{1,3^{*}+}$ \\ ${ }^{1}$ Department of Psychological and Brain Sciences, University of California, Santa Barbara \\ ${ }^{2}$ Department of Neurobiology and Behavior, University of California, Irvine \\ ${ }^{3}$ Neuroscience Research Institute, University of California, Santa Barbara \\ ${ }^{4}$ Department of Geography, University of California, Santa Barbara
}

*Corresponding Authors

$\uparrow$ Denotes equal contributions of senior authors

+Now at the School of Psychological Science, Oregon State University

\section{Please address comments to:}

Elizabeth R. Chrastil, Ph.D.

Department of Neurobiology and Behavior

University of California, Irvine

chrastil@uci.edu

Mary Hegarty, Ph.D.

Department of Psychological and Brain Sciences

University of California, Santa Barbara

hegarty@ucsb.edu

Emily G. Jacobs, Ph.D.

Department of Psychological and Brain Sciences

University of California, Santa Barbara

emily.jacobs@psych.ucsb.edu 


\begin{abstract}
Accumulating evidence suggests that distinct aspects of successful navigation—path integration, acquiring spatial knowledge, and navigation strategies—change with advanced age. Yet, few studies have established whether navigation deficits emerge early in the aging process (prior to age 65) or whether early age-related deficits vary by sex. Here, we probed healthy young (ages 18-28) and midlife (ages 43-61) adults on three essential aspects of navigation. First, path integration ability shows negligible effects of sex or age. Second, robust sex differences in spatial knowledge acquisition are observed in young adulthood and persist, but are diminished, with age. Third, by midlife, men and women show decreased ability to acquire spatial knowledge and increased reliance on taking habitual paths. Together, our findings indicate that age-related changes in navigation ability and strategy are evident as early as midlife and that path integration ability is relatively spared in the transition from youth to middle age.
\end{abstract}

Key Words: cognitive aging | virtual reality | path integration | wayfinding 


\section{Statement of Relevance}

Few studies have established whether navigation deficits emerge early in the aging process (prior to age 65) or whether early age-related deficits vary by sex. This represents a critical gap in our understanding of the aging brain, especially given known changes in navigation-related brain regions during the menopausal transition. Here, we examined signatures of early aging in three navigational tasks, opening up new avenues for understanding healthy aging. Although path integration has typically been used as an early marker for dementia, our findings suggest that spatial knowledge acquisition and strategy use are more sensitive to the earliest stages of the aging process. Moving forward, using a comprehensive assessment of spatial navigation may offer a more sensitive behavioral fingerprint of a person's dementia risk. Understanding the trajectories of healthy aging — and how they differ for men and women—will pave the way for developing behavioral and neural markers for dementia. 


\section{Age-related changes in spatial navigation are evident by midlife and differ by sex}

Spatial navigation refers to processes by which we update our position and orientation in space, learn the layout of new places, and plan routes to goal locations in known environments. Successful navigation is a complex behavior, requiring the integration of multiple perceptual cues, memory, and executive processes (Chrastil, 2013; Hegarty et al., 2006; Wolbers \& Hegarty, 2010). Despite the importance of navigation to our daily lives, there are large individual and sex differences in navigation ability (Hegarty et al., 2002, 2006; Ishikawa \& Montello, 2006; Nazareth et al., 2019; Weisberg \& Newcombe, 2016), and deficits in navigational abilities are apparent in older adult (ages 65+) populations (Harris \& Wolbers, 2012; Lester et al., 2017; Merhav \& Wolbers, 2019; Zhong \& Moffat, 2016). However, little is known about when these changes emerge during the aging process, or whether sex differences evident in younger populations persist with age. This represents a critical gap in our understanding of the aging brain.

Spatial navigation has emerged as a promising behavioral marker for detecting individuals at risk for dementia. Young adults with a heightened genetic risk for Alzheimer's disease $(\mathrm{AD})$ have poorer navigation performance and altered neural activity during navigation, decades before the onset of disease symptoms (Coughlan et al., 2018a, 2018b; Kunz et al., 2015). The prevalence of $\mathrm{AD}$ is two-fold greater in women than in men (Lin et al., 2015), suggesting sex plays a role in disease risk. An emerging consensus is that the sweeping neuroendocrine changes that occur during the midlife transition to menopause are a sex-specific risk factor for AD. Notably, the brain regions most sensitive to changes in sex hormone production are the hippocampal, entorhinal, and prefrontal cortices (Jacobs \& Goldstein, 2018)—key regions within the brain's navigational circuitry (Lester et al., 2017; Moffat, 2009; Zhong \& Moffat, 2016). 
Together, these findings suggest that taking into account sex differences in age-related changes in navigational ability could be critical for the early detection of individuals at risk for neurodegenerative disease.

In the present study, we probed healthy young (ages 18-28) and midlife (ages 43-61) men and women on three essential aspects of navigation: path integration, spatial knowledge acquisition, and navigational strategy (Figure 1). This design allows us to gain a comprehensive understanding of when navigational deficits emerge in the aging process in men and women.

Path integration is the updating of one's position and orientation during self-motion without external landmarks, and relies on internal senses of self-motion from proprioceptive and vestibular systems, as well as visual information from optic flow (Loomis et al., 1993; Mittelstaedt \& Mittelstaedt, 1980). Older adults (65+) are impaired on path integration (Allen et al., 2004; Harris \& Wolbers, 2012; Stangl et al., 2018), but it is unknown if these deficits are present earlier in the aging process. Further, it is unclear whether sex differences in path integration exist at any point across the lifespan (Coughlan et al., 2018a; Coutrot et al., 2019).

Here, we tested path integration ability with a walking virtual reality (VR) task (Chrastil et al., 2019). The deficits in path integration observed in older adults (Allen et al., 2004; Harris \& Wolbers, 2012; Stangl et al., 2018) were based on studies that provided only proprioception or only vision (e.g., using desktop virtual environments or blindfolded walking). In contrast, our task provides participants with both cues. Based on previous research, we might expect agerelated deficits to emerge as early as midlife. However, age related deficits in path integration might be spared when consistent cues from vision and proprioception are available. Sex differences are prevalent in the ability to form survey knowledge (Chrastil \& Warren, 2013; Moffat et al., 1998; Nazareth et al., 2019; Waller, 2000), which is presumed to rely on path 
integration, so we might expect to observe a sex difference in path integration. However, to date no direct test of sex differences in path integration has been performed using proprioceptive and visual cues, which represents a novel aspect of our study.

Spatial knowledge acquisition (sometimes referred to as cognitive mapping) is the ability to acquire spatial information such as inferring how paths connect and where items are located in the broader environment (Golledge, 1999). Sex differences in spatial knowledge acquisition from both route learning and free exploration have been observed in young adults (Chrastil \& Warren, 2015; Coluccia \& Louse, 2004; Hegarty et al., 2006; Montello et al., 1999). However, few studies have examined spatial knowledge acquisition from free exploration as opposed to route learning (e.g., Wiener et al., 2013) in older adults. Zhong \& Moffat (2016) allowed participants to discover the correct route to a goal location, finding both sex and aging effects across young, midlife, and older populations. However, the effects of aging on the ability to learn a spatial layout from unrestricted exploration are still unknown.

We tested spatial knowledge acquisition using a desktop VR maze task (Chrastil \& Warren, 2015). Critically, participants were free to explore the maze, a novel paradigm for testing aging populations. We expect sex differences in spatial knowledge acquisition for young adults (cf. Chrastil \& Warren, 2015). However, while age effects have been observed in route learning in older adults ages 65+ (Harris et al., 2012; Wiener et al., 2013; Zhong \& Moffat, 2016), it is unclear whether unrestricted learning also leads to deficits in older adults. We also examined unrestricted exploration behavior to disambiguate whether deficits arise from a failure to fully explore a novel spatial environment or from a failure to consolidate exposure into spatial knowledge. Because the neuroendocrine changes during menopause affect the circuitry for 
acquiring spatial knowledge (Jacobs \& Goldstein, 2018), we predicted a male advantage would persist with age.

Navigational strategies refer to the nature of the paths individuals select to navigate to a goal location in a known environment. Sex differences in navigation strategy have been observed in young adults (Boone et al., 2018), with women more likely to follow well-learned (habitual) routes using cues from landmarks (an egocentric, "response-based" strategy), whereas men more often use their knowledge of the spatial layout of an environment to infer a shortcut to a goal location (an allocentric, "place-based" strategy). Older adults (65+) are more likely to follow established routes rather than take novel shortcuts compared to younger adults (Harris \& Wolbers, 2014) and are less flexible at switching between response and place-based strategies (Harris et al., 2012; Wiener et al., 2013). Despite accumulating evidence of navigation deficits in elderly populations, navigational strategies have yet to be examined earlier in the aging process, another critical gap in our understanding of the aging brain.

We had participants learn a set route in a desktop VR environment (Marchette et al., 2011), and then probed their navigational strategies by examining the paths they took when asked to navigate to target locations in the maze. This study is the first to investigate sex differences in strategy preference in midlife adults. On the basis of previous research with older adults, we hypothesized that midlife adults would show an increased reliance on habitual paths relative to young adults and that men would display a greater preference for taking novel shortcuts compared to women.

In sum, we probed three essential aspects of spatial navigation to determine whether behavioral deficits are detectable in the early stages of the aging process. Characterizing these 
changes in the healthy aging brain, and how they differ for men and women, is critical to future work establishing the earliest behavioral signs of AD and other dementias.

\section{Method}

\section{Participants}

One hundred and fifty-one adults from the University of California, Santa Barbara and greater Santa Barbara community participated in the study. These included eighty-five young adults (39 females, age range $18-28, M=19.81, S D= \pm 1.87$ ) and sixty-six midlife adults (40 females, age range 43-61, $M=51.14, S D= \pm 4.07)$. Participants gave written informed consent and the study was approved by the University of California, Santa Barbara Human Subjects Committee. Young adults were compensated financially (\$12/hour) or through course credit. Midlife participants were compensated financially ( $\$ 30 /$ hour to account for the additional costs of traveling from off-campus).

Due to technical issues with the equipment and simulator motion sickness, some participants could not complete all three tasks or were excluded from data analysis because of insufficient trials. Thus, final sample sizes are reported for each analysis and reported in each corresponding figure. A power analysis was performed for sample size estimation based on our 2 (age group) $\times 2($ sex $)$ design. To detect a medium-to-large effect size $(f=.30)$ using Cohen's criteria (1988) with an alpha $=.05$ and $80 \%$ power, the projected sample size needed is $N=90$ participants. Thus, our sample should be sufficiently powered to test effects of age and sex.

\{Insert Figure 1 here\}

\section{Task Procedures}


Participants completed three VR-based navigation tasks to assess path integration, spatial knowledge, and navigational strategy (Figure 1).

\section{Loop Closure Task}

The Loop Closure Task ("LOOP") is an immersive, walking VR paradigm that assesses path integration ability (Figure 1, Task 1). A detailed description of the methods has been reported previously (Chrastil et al., 2019). Briefly, participants saw a bare desert landscape devoid of landmarks or other orienting cues. Visual (optic flow), proprioceptive, and vestibular information was available to participants. An orange pole served as the start location for each

trial, but it was not visible after the trial commenced. Participants walked along a circular loop, while an experimenter guided them to ensure they stayed on the circumference of the circle. Participants clicked a button on a wireless remote to indicate when they thought they had returned to the start location. Participants completed 10 trials per radius, with $1.0 \mathrm{~m}, 2.0 \mathrm{~m}$, and 3.0m loops. Performance feedback was not provided and there was no time limit to complete each trial.

\section{\{Insert Figure 2 here\}}

The primary dependent variables were position error and total degrees traveled (Figure 2A). Position error is defined as the straight line distance between the actual starting location for each trial and the location that the participant indicated was the start. Total degrees traveled represents the number of degrees traveled around the circular path (which can be greater than, less than, or equal to $360^{\circ}$ ). Variability of position error and degrees traveled, defined as the within-subjects standard deviation across the 10 trials at each radius, was also assessed. Variability across trials could indicate how well the participants were able to integrate the cues 
on a consistent basis; high variability could indicate less certainty in the integration (Chrastil et al., 2019). A mixed model ANOVA was conducted for the four outcome measures with a 2 (age group: midlife, young $) \times 2($ sex: female, male $) \times 3$ (radius size: $1.0 \mathrm{~m}, 2.0 \mathrm{~m}, 3.0 \mathrm{~m})$ design . Corrections for sphericity were made where appropriate using the Greenhouse-Geisser correction.

\section{MAZE Learning Task}

The MAZE Learning Task ("MAZE”) assesses an individual's ability to acquire spatial knowledge. Details of the task have been reported previously (Chrastil \& Warren, 2014, 2015), with the exception that, in this case, the MAZE task was conducted with a desktop VR setup. Briefly, the virtual maze environment consists of hallways with tall, vertical hedges and nine target objects. During an initial exploration phase, participants were given two 8-minute sessions to freely navigate the virtual environment with the goal of finding the nine target objects (indicated as red dots in the maze depicted in Figure 1, Task 2). Paintings served as landmarks to guide and orient participants (indicated as purple rectangles in Figure 1, Task 2). Participants used the computer keyboard to indicate at each intersection whether they wanted to move left, right, or straight. Translational movement was fixed at $1.0 \mathrm{~m} / \mathrm{s}$ (virtual meters $/$ second) and rotation speed fixed at $90^{\circ}$ per second. During the test phase, participants completed 24 trials to assess their spatial knowledge of the environment. Each trial began with the participant located at one object in the maze and given the instruction to navigate to another object within a 45 second trial period. The objects were replaced with red spheres during the test phase to minimize feedback and landmark cues during navigation.

The main dependent variable was wayfinding success (a measure of spatial knowledge acquisition based on free exploration), defined as the proportion of trials in which the participant 
reached the correct target object in time during the test phase. This variable was compared against chance (i.e., $1 / 9$ potential objects in the maze $=11.11 \%$ ). We also measured the total number of moves - key presses made to navigate the environment during the exploration phaseas a measure of how extensively participants explored the maze, to determine how exploration relates to test performance. A 2 (age group: midlife, young) $\times 2$ (sex: female, male) betweensubjects ANOVA was conducted to assess group differences in each of these variables. Finally, a Pearson's correlation assessed the relationship between the number of moves made during the exploration phase and navigation success in the test phase. An analysis of covariance (ANCOVA) examined the effects of age and sex on navigation success, after controlling for the number of moves made by participants during maze exploration.

\section{Dual-solution Paradigm}

The Dual-solution Paradigm ("DSP") assesses individual differences in navigational strategy. Procedures were identical to those used by Boone et al., 2018. Participants first familiarized themselves with the navigation controls in an open desktop VR training maze, prior to the task. The DSP maze consisted of an environment with 12 landmarks (shown as black diamonds in Figure 1, Task 3). In contrast to the MAZE task, in which participants were free to explore the environment during the initial learning phase, all participants were given the same learning experience in the DSP. Specifically, they were guided along the same route through the maze 5 times (depicted as a purple path starting at the red dot in Figure 1, Task 3). Then during the test phase, participants were placed at one object in the maze and instructed to navigate to another object. All objects were visible throughout the test phase. Participants had 40 seconds to find each target object and completed a total of 20 trials. Trials were coded to determine whether participants took the learned route or a novel shortcut when navigating to the target object. Trials 
were chosen such that the shortest route to the target was at least $25 \%$ shorter than the learned route; shortcuts were on average, 51\% shorter. Participants had to take the shortest possible route to count as a shortcut (for additional details on trial coding, see: Supplemental Material and Boone et al., 2018, 2019).

Two measures of interest were calculated: wayfinding success (a measure of spatial knowledge acquisition based on route experience), defined as the proportion of trials on which the participant reached the target object within the time limit, and the solution index, defined as the number of shortcuts (the shortest possible route) divided by the number of successful trials (a measure of strategy). A 2 (age group: midlife, young) $\times 2$ (sex: female, male) between-subjects ANOVA was performed on each dependent variable. Heatmaps were generated to provide a qualitative assessment of participants' routes in the virtual environment by extracting their location every $100 \mathrm{~ms}$ per trial.

\section{Results}

\section{Loop Closure Task}

Fifty-two young adults ( 25 females, 27 males) and fifty-six midlife adults (33 females, 23 males) were included in the data analysis. Participants' data were removed if insufficient trials ( $n$ $<5$ trials) were completed at a given radius, resulting in 105 data points for radius $1.0 \mathrm{~m}, 108$ for radius 2.0m, and 90 for radius 3.0m (see Supplemental Material).

We first tested the prediction that there would be age and sex differences in path integration accuracy. Position error (Figure 2A) did not differ significantly as a function of age $\left(F_{1,85}=.92, p=.341, \eta_{p}^{2}=.01\right)$ or $\operatorname{sex}\left(F_{1,85}=2.84, p=.096, \eta_{p}{ }^{2}=.03\right)$. As expected, position error increased with radius size $\left(F_{2,170}=328.57, p<.001, \eta_{p}^{2}=.80\right.$; Figure 2B), suggesting that 
path integration is more difficult with longer distances. Although there was a significant age $\times$ radius size interaction $\left(F_{2,170}=4.15, p=.003, \eta_{p}{ }^{2}=.05\right)$ and sex $\times$ radius size interaction $\left(F_{2,170}\right.$ $\left.=7.13, p=.003, \eta_{p}{ }^{2}=.08\right)$, post-hoc testing found no significant effects of sex or age at any level of radius (all $p>.060$, Bonferroni corrected). Further, no age $\times$ sex interaction $\left(F_{1,85}=\right.$ $\left.1.21, p=.274, \eta_{p}^{2}=.01\right)$ or three-way interaction $\left(F_{2,170}=2.68, p=.086, \eta_{p}{ }^{2}=.03\right)$ was observed.

Variability in position error showed no main effects of age $\left(F_{1,85}=.45, p=.502, \eta_{p}^{2}=\right.$ $.005)$ or $\operatorname{sex}\left(F_{1,85}=.08, p=.779, \eta_{p}^{2}<.001\right)$. As shown in Figure 2C, there was a main effect of radius size $\left(F_{2,170}=452.01, p<.001, \eta_{p}^{2}=.84\right)$, with increasing radius size leading to increased variability in participants' estimates. There were no significant interactions for this variable (all $p>.133)$.

Degrees traveled around the circular loop path did not differ significantly as a function of age $\left(F_{1,85}=.61, p=.437, \eta_{p}^{2}=.01\right)$. However, there was a significant main effect of $\operatorname{sex}\left(F_{1,85}=\right.$ 5.82, $p=.018, \eta_{p}{ }^{2}=.06$ ) as shown in Figure 2D, such that women tended to overshoot their estimate of the starting location, while men tended to undershoot. A main effect of radius size $\left(F_{2,170}=9.33, p<.001, \eta_{p}{ }^{2}=.10\right)$, indicated that degrees traveled increased as the size of the radius increased. There were no significant interactions between these variables (all $p>.144$ ).

Variability in degrees traveled also showed no significant main effects of age $\left(F_{1,85}=.01\right.$, $\left.p=.929, \eta_{p}^{2}<.001\right)$ or $\operatorname{sex}\left(F_{1,85}=1.67, p=.200, \eta_{p}^{2}=.02\right)$, but there was a main effect of radius size $\left(F_{2,170}=9.29, p<.001, \eta_{p}^{2}=.10\right)$. Variability increased as the size of the radius increased. None of the interactions for variability in degrees traveled were significant (all $p>$ .083) (Figure 2E). 
In summary, position error was similar across young and middle-aged men and women, while degrees traveled showed that women tended to overshoot while men tended to undershoot. Overall, performance accuracy for the LOOP task suggests negligible effects of age and sex on path integration ability.

\section{MAZE Learning Task}

Fifty young adults (26 females, 24 males) and thirty-nine midlife adults ( 25 females, 14 males) were included in the data analysis. First, we tested whether participants performed above chance. Young men $\left(t_{23}=8.72, p<.001\right)$ and women $\left(t_{25}=3.38, p=.001\right)$ were significantly above chance, while midlife men $\left(t_{13}=1.86, p=.043\right)$ and women $\left(t_{24}=-1.27, p=.892\right)$ were close to or at chance performance. Next, we tested the prediction that there would be age and sex differences in wayfinding success during the test phase. There was a significant main effect of age $\left(F_{1,85}=30.37, p<.001, \eta_{p}^{2}=.26\right)$, with better performance by younger adults than midlife adults, and a significant effect of $\operatorname{sex}\left(F_{1,85}=39.86, p<.001, \eta_{p}{ }^{2}=.32\right)$, with men having more wayfinding success than women. There was also a significant age $\times$ sex interaction $\left(F_{1,85}=8.04\right.$, $\left.p=.006, \eta_{p}{ }^{2}=.09\right)$. An analysis of simple effects revealed significant effects of age on both sexes (men: $F_{1,36}=15.65, p<.001, \eta_{p}{ }^{2}=.30$; women: $\left.F_{1,49}=12.49, p=.002, \eta_{p}{ }^{2}=.20\right)$. Simple effects also indicated significant effects of sex at both ages, with substantially larger effects for young $\left(F_{1,48}=41.25, p<.001, \eta_{p}^{2}=.46\right)$ than for midlife adults $\left(F_{1,37}=7.18, p=.022, \eta_{p}^{2}=.16\right)$. While sex differences were reduced in middle aged compared to younger adults, it should be noted that performance was generally very poor among midlife adults, so this could partially reflect a floor effect.

During the initial maze exploration phase, participants made on average $268.08 \pm 39.94$ moves to explore the maze. Across all participants, a significant Pearson's correlation test $(r(87)$ 
$=.34, p=.001)$ indicated that wayfinding success was related to how much participants explored the maze. As shown in Figure 3B, a 2 (age group) $\times 2$ (sex) ANOVA indicated a main effect of age $\left(F_{1,85}=26.99, p<.001, \eta_{p}^{2}=.24\right)$, with younger adults making more moves. There was no main effect of $\operatorname{sex}\left(F_{1,85}=1.51, p=.223, \eta_{p}^{2}=.02\right)$, or an age $\times$ sex interaction $\left(F_{1,85}=.57, p=\right.$ $\left..453, \eta_{p}^{2}=.01\right)$ for number of moves made during exploration.

Due to this difference in moves across age groups, we conducted a two-way ANCOVA on wayfinding success to test whether the differences in moves made between age groups during the exploration phase affected performance in the test phase. After controlling for moves made, the main effects of age $\left(F_{1,84}=18.51, p<.001, \eta_{p}{ }^{2}=.18\right)$ and $\operatorname{sex}\left(F_{1,84}=37.52, p<.001, \eta_{p}{ }^{2}=\right.$ .31) remained significant, as did the two-way interaction between age and sex $\left(F_{1,84}=8.46, p=\right.$ $\left..005, \eta_{p}^{2}=.09\right)$. An analysis of simple effects of age remained significant for men $\left(F_{1,35}=11.30\right.$, $\left.p=.008, \eta_{p}{ }^{2}=.24\right)$ but not women $\left(F_{1,48}=4.86, p=.128, \eta_{p}{ }^{2}=.09\right)$. The simple effect of sex remained statistically significant for young adults $\left(F_{1,47}=40.57, p<.001, \eta_{p}{ }^{2}=.46\right)$, but not for midlife adults $\left(F_{1,36}=5.46, p=.100, \eta_{p}{ }^{2}=.13\right)$, as shown in Figure 3A.

In summary, men showed a steep age-related deficit in wayfinding success. The sex difference favoring men in young adulthood was eliminated by midlife.

\section{\{Insert Figure 3 here\}}

\section{Dual-solution Paradigm}

Fifty-four young adults (26 females, 28 males) and forty midlife adults (19 females, 21 males) were included in the data analysis. We first tested the prediction that young adults would outperform midlife adults on wayfinding success. We observed a main effect of age $\left(F_{1,90}=\right.$ 43.04, $p<.001, \eta_{p}^{2}=.32$ ), with younger participants outperforming midlife participants, and a 
main effect of $\operatorname{sex}\left(F_{1,90}=15.95, p<.001, \eta_{p}{ }^{2}=.15\right)$, with men outperforming women (Figure 4A), but with no age $\times$ sex interaction $\left(F_{1,90}=.38, p=.537, \eta_{p}^{2}=.004\right)$.

\section{\{Insert Figure 4 here\}}

We then tested the prediction that younger adults would take more shortcuts on successful trials (i.e., have a higher solution index) than midlife adults. We observed a main effect of age $\left(F_{1,90}=7.83, p=.006, \eta_{p}^{2}=.08\right)$, with younger adults taking more shortcuts than midlife adults. We observed a marginally significant main effect of $\operatorname{sex}\left(F_{1,90}=3.64, p=.06, \eta_{p}{ }^{2}\right.$ $=.04$ ), with men tending to take more shortcuts than women. However, there was a significant age $\times$ sex interaction $\left(F_{1,90}=6.59, p=.012, \eta_{p}{ }^{2}=.07\right)($ Figure 4B). An analysis of simple effects revealed that the effect of age was significant for men $\left(F_{1,47}=14.00, p<.001, \eta_{p}{ }^{2}=.23\right)$ but not for women $\left(F_{1,43}=.03, p=1.00, \eta_{p}^{2}<.001\right)$. Simple effects also indicated significant effects of sex for young adults $\left(F_{1,52}=10.47, p=.004, \eta_{p}^{2}=.17\right)$ but not for midlife adults $\left(F_{1,38}\right.$ $\left.=.23, p=1.00, \eta_{p}{ }^{2}=.01\right)$. Heatmaps were generated as a qualitative visualization of the routes people chose to reach a target (Figure 5) and indicate that young men were more likely to take a shortcut through the center of the maze, while young women, midlife men, and midlife women favored the learned route along the periphery.

\section{\{Insert Figure 5 here\}}

\section{Discussion}

The present study tested three core aspects of spatial navigation in young and midlife adults: path integration, spatial knowledge acquisition, and navigational strategy. Building on previous chronological aging studies, our findings reveal that some age-related differences in spatial navigation are already evident by midlife. While path integration ability was largely 
preserved with age in the LOOP task, pronounced age-related differences were observed in the ability to acquire spatial knowledge in the MAZE task and in the selection of a navigational strategy in the DSP task. Thus, no major sex difference was observed for path integration, while sex differences were found for acquiring spatial knowledge and navigation strategy. Overall, sex differences present in young adults tended to be reduced with age.

Previous findings indicate poor path integration in older adults aged 65+ (Adamo et al., 2012; Coughlan et al., 2018a; Harris \& Wolbers, 2012), however we saw no such deficit in midlife adults, suggesting that age-related deficits arise later in the aging process. In previous studies older adults had access to a single cue (vision or proprioception), whilst our task provided participants with multiple cues. Future studies should examine whether performance deficits are evident in midlife when performance is constrained to a single cue. Although there was a sex difference in degrees traveled, with women tending to overshoot and men tending to undershoot, this difference did not affect the overall position error. The tendency to overshoot could be a cautionary measure by women to ensure they reach the start (e.g., Gagnon et al., 2016). In sum, we found little evidence for sex or aging effects in path integration.

Sex differences were evident in measures of spatial knowledge acquisition from both open exploration (MAZE task) and route-based learning (DSP task). For the MAZE task, midlife adults did not explore as much as younger adults. After accounting for differing numbers of exploration moves, sex differences remained robust in young adults and diminished in midlife adults. Floor effects were present in the MAZE task for midlife adults, making sex differences less detectible. It is possible that age-related changes in the brain hinder the ability for midlife adults to create a comprehensive cognitive map, which in turn make sex differences in performance harder to detect. In the DSP, wayfinding success indicates how well participants 
learned the environment from a route. Despite equal exposure to the route, midlife adults were less successful than younger adults, consistent with previous studies on learning from routes (Harris \& Wolbers, 2014; Wiener et al., 2013; Zhong \& Moffat, 2016). Together, these finding suggest spatial learning is impaired as early as midlife.

Navigation strategies in the DSP indicated that young men took more shortcuts than young women, echoing previous results by Boone et al. ( 2018; 2019). Our study provides the first evidence that age-related differences in navigation strategies are evident by midlife, with midlife adults using fewer shortcuts than younger adults. This result is consistent with reports that older adults use more habitual routes when navigating (Harris et al., 2012; Lester et al., 2017; Wiener et al., 2013), and suggests that strategies have already shifted by midlife. In addition, we found that the sex difference observed in young adults did not persist in midlife adults. Further, the heat maps indicated that while young men were more likely to take shortcuts through the middle of the maze, all other groups relied on the learned route to navigate within the maze. Thus, the major change with age was a reduction of place-based strategies in men.

One limitation is the possibility that older adults have less experience with using typical computer gaming controls (as used in the DSP task) compared to younger adults, and this contributed to navigation inefficiency in the desktop virtual environments. However, age-related differences also existed for the MAZE task, but the desktop VR in that task relied much less on the use of a gaming interface: it used a single button press at each intersection. This indicates that poorer performance for midlife adults is unlikely to be due to computer experience alone, and likely to be related to age-related changes in the brain that deterred successful acquisition of spatial information from the environment. 
In sum, we examined signatures of early aging in three navigational tasks, opening up new avenues for understanding healthy aging. The differing patterns of age and sex across our three navigational tasks suggest that different aspects of navigation could tap into separate brain systems. Although path integration has typically been used as an early marker for dementia (Coughlan et al., 2018b; Kunz et al., 2015), our findings suggest that spatial knowledge acquisition and strategy use are more sensitive to the earliest stages of the aging process. Understanding the trajectories of healthy aging — and how they differ for men and women—will help pave the way for developing behavioral and neural markers for dementia.

Author Contributions: M. Hegarty, E.R. Chrastil, and E.G. Jacobs developed the study concept. S. Yu and R.C. Davis performed the experiments. Data analysis concept was developed by S. Yu, A.P. Boone, C. He, M. Hegarty, E.R. Chrastil, E.G. Jacobs, and S. Yu performed the data analysis. S. Yu drafted the manuscript, and M. Hegarty, E.R. Chrastil, and E.G. Jacobs provided critical revisions. A.P. Boone, C. He, and R.C. Davis edited the manuscript.

Acknowledgements: This work was supported by the California Nanosystems Institute (EGJ, ERC, MH), the Brain and Behavior Research Foundation (EGJ), the Institute for Collaborative Biotechnologies (ERC), the UCSB Academic Senate (EGJ, ERC, MH), and the Hellman Fellows Fund (EGJ). We would like to thank Mustafa Shakir, Julia Woods, Chloe Lopez, and Aidan Galati for assistance with data collection and coding. 


\section{References}

Adamo, D. E., Briceño, E. M., Sindone, J. A., Alexander, N. B., \& Moffat, S. (2012). Age differences in virtual environment and real world path integration. Frontiers in Aging Neuroscience, 4. https://doi.org/10.3389/fnagi.2012.00026

Allen, G. L., Kirasic, K. C., Rashotte, M. A., \& Haun, D. B. M. (2004). Aging and path integration skill: Kinesthetic and vestibular contributions to wayfinding. Perception \& Psychophysics, 66(1), 170-179. https://doi.org/10.3758/BF03194870

Boone, A. P., Gong, X., \& Hegarty, M. (2018). Sex differences in navigation strategy and efficiency. Memory \& Cognition, 46(6), 909-922. https://doi.org/10.3758/s13421-0180811-y

Boone, A. P., Maghen, B., \& Hegarty, M. (2019). Instructions matter: Individual differences in navigation strategy and ability. Memory \& Cognition, 47(7), 1401-1414. https://doi.org/10.3758/s13421-019-00941-5

Chrastil, E. R. (2013). Neural evidence supports a novel framework for spatial navigation. Psychonomic Bulletin \& Review, 20(2), 208-227. https://doi.org/10.3758/s13423-012$0351-6$

Chrastil, E. R., Nicora, G. L., \& Huang, A. (2019). Vision and proprioception make equal contributions to path integration in a novel homing task. Cognition, 192, 103998. https://doi.org/10.1016/j.cognition.2019.06.010

Chrastil, E. R., \& Warren, W. H. (2013). Active and passive spatial learning in human navigation: Acquisition of survey knowledge. Journal of Experimental Psychology: Learning, Memory, and Cognition, 39(5), 1520-1537. http://dx.doi.org.proxy.library.ucsb.edu:2048/10.1037/a0032382 
Chrastil, E. R., \& Warren, W. H. (2014). From Cognitive Maps to Cognitive Graphs. PLOS ONE, 9(11), e112544. https://doi.org/10.1371/journal.pone.0112544

Chrastil, E. R., \& Warren, W. H. (2015). Active and passive spatial learning in human navigation: Acquisition of graph knowledge. Journal of Experimental Psychology: Learning, Memory, and Cognition, 41(4), 1162-1178. https://doi.org/10.1037/xlm0000082

Cohen, J. (1988). Statistical Power Analysis for the Behavioral Sciences (2nd ed.). Lawrence Erlbaum Associates, Publishers.

Coluccia, E., \& Louse, G. (2004). Gender differences in spatial orientation: A review. Journal of Environmental Psychology, 24(3), 329-340. https://doi.org/10.1016/j.jenvp.2004.08.006

Coughlan, G., Coutrot, A., Khondoker, M., Minihane, A.-M., Spiers, H. J., \& Hornberger, M. (2018). Impact of Sex and APOE Status on Spatial Navigation in Pre-symptomatic Alzheimer's disease. BioRxiv, 287722. https://doi.org/10.1101/287722

Coughlan, G., Laczó, J., Hort, J., Minihane, A.-M., \& Hornberger, M. (2018). Spatial navigation deficits-Overlooked cognitive marker for preclinical Alzheimer disease? Nature Reviews Neurology, 14(8), 496-506. https://doi.org/10.1038/s41582-018-0031-x

Coutrot, A., Schmidt, S., Coutrot, L., Pittman, J., Hong, L., Wiener, J. M., Hölscher, C., Dalton, R. C., Hornberger, M., \& Spiers, H. J. (2019). Virtual navigation tested on a mobile app is predictive of real-world wayfinding navigation performance. PLOS ONE, 14(3), e0213272. https://doi.org/10.1371/journal.pone.0213272

Gagnon, K. T., Cashdan, E. A., Stefanucci, J. K., \& Creem-Regehr, S. H. (2016). Sex Differences in Exploration Behavior and the Relationship to Harm Avoidance. Human Nature, 27(1), 82-97. https://doi.org/10.1007/s12110-015-9248-1 
Golledge, R. G. (1999). Wayfinding Behavior: Cognitive Mapping and Other Spatial Processes. JHU Press.

Harris, M. A., Wiener, J. M., \& Wolbers, T. (2012). Aging specifically impairs switching to an allocentric navigational strategy. Frontiers in Aging Neuroscience, 4. https://doi.org/10.3389/fnagi.2012.00029

Harris, M. A., \& Wolbers, T. (2012). Ageing effects on path integration and landmark navigation. Hippocampus, 22(8), 1770-1780. https://doi.org/10.1002/hipo.22011

Harris, M. A., \& Wolbers, T. (2014). How age-related strategy switching deficits affect wayfinding in complex environments. Neurobiology of Aging, 35(5), 1095-1102. https://doi.org/10.1016/j.neurobiolaging.2013.10.086

Hegarty, M., Montello, D. R., Richardson, A. E., Ishikawa, T., \& Lovelace, K. (2006). Spatial abilities at different scales: Individual differences in aptitude-test performance and spatial-layout learning. Intelligence, 34(2), 151-176. https://doi.org/10.1016/j.intell.2005.09.005

Hegarty, M., Richardson, A. E., Montello, D. R., Lovelace, K., \& Subbiah, I. (2002). Development of a self-report measure of environmental spatial ability. Intelligence, 30(5), 425-447. https://doi.org/10.1016/S0160-2896(02)00116-2

Ishikawa, T., \& Montello, D. R. (2006). Spatial knowledge acquisition from direct experience in the environment: Individual differences in the development of metric knowledge and the integration of separately learned places. Cognitive Psychology, 52(2), 93-129. https://doi.org/10.1016/j.cogpsych.2005.08.003 
Jacobs, E. G., \& Goldstein, J. M. (2018). The middle-aged brain: Biological sex and sex hormones shape memory circuitry. Current Opinion in Behavioral Sciences, 23, 84-91. https://doi.org/10.1016/j.cobeha.2018.03.009

Kunz, L., Schröder, T. N., Lee, H., Montag, C., Lachmann, B., Sariyska, R., Reuter, M., Stirnberg, R., Stöcker, T., Messing-Floeter, P. C., Fell, J., Doeller, C. F., \& Axmacher, N. (2015). Reduced grid-cell-like representations in adults at genetic risk for Alzheimer's disease. Science, 350(6259), 430-433. https://doi.org/10.1126/science.aac8128

Lester, A. W., Moffat, S. D., Wiener, J. M., Barnes, C. A., \& Wolbers, T. (2017). The Aging Navigational System. Neuron, 95(5), 1019-1035. https://doi.org/10.1016/j.neuron.2017.06.037

Lin, K. A., Choudhury, K. R., Rathakrishnan, B. G., Marks, D. M., Petrella, J. R., \& Doraiswamy, P. M. (2015). Marked gender differences in progression of mild cognitive impairment over 8 years. Alzheimer's \& Dementia: Translational Research \& Clinical Interventions, 1(2), 103-110. https://doi.org/10.1016/j.trci.2015.07.001

Loomis, J. M., Klatzky, R. L., Golledge, R. G., Cicinelli, J. G., Pellegrino, J. W., \& Fry, P. A. (1993). Nonvisual navigation by blind and sighted: Assessment of path integration ability. Journal of Experimental Psychology: General, 122(1), 73-91. https://doi.org/10.1037/0096-3445.122.1.73

Marchette, S. A., Bakker, A., \& Shelton, A. L. (2011). Cognitive Mappers to Creatures of Habit: Differential Engagement of Place and Response Learning Mechanisms Predicts Human Navigational Behavior. Journal of Neuroscience, 31(43), 15264-15268. https://doi.org/10.1523/JNEUROSCI.3634-11.2011 
Merhav, M., \& Wolbers, T. (2019). Aging and spatial cues influence the updating of navigational memories. Scientific Reports, 9(1), 1-10. https://doi.org/10.1038/s41598-019-47971-2

Mittelstaedt, M.-L., \& Mittelstaedt, H. (1980). Homing by path integration in a mammal. Naturwissenschaften, 67(11), 566-567. https://doi.org/10.1007/BF00450672

Moffat, S. D. (2009). Aging and Spatial Navigation: What Do We Know and Where Do We Go? Neuropsychology Review, 19(4), 478. https://doi.org/10.1007/s11065-009-9120-3

Moffat, S. D., Hampson, E., \& Hatzipantelis, M. (1998). Navigation in a "Virtual" Maze: Sex Differences and Correlation With Psychometric Measures of Spatial Ability in Humans. Evolution and Human Behavior, 19(2), 73-87. https://doi.org/10.1016/S10905138(97)00104-9

Montello, D. R., Lovelace, K. L., Golledge, R. G., \& Self, C. M. (1999). Sex-Related Differences and Similarities in Geographic and Environmental Spatial Abilities. Annals of the Association of American Geographers, 89(3), 515-534. https://doi.org/10.1111/0004-5608.00160

Nazareth, A., Huang, X., Voyer, D., \& Newcombe, N. (2019). A meta-analysis of sex differences in human navigation skills. Psychonomic Bulletin \& Review, 26(5), 1503-1528. https://doi.org/10.3758/s13423-019-01633-6

Stangl, M., Achtzehn, J., Huber, K., Dietrich, C., Tempelmann, C., \& Wolbers, T. (2018). Compromised Grid-Cell-like Representations in Old Age as a Key Mechanism to Explain Age-Related Navigational Deficits. Current Biology, 28(7), 1108-1115.e6. https://doi.org/10.1016/j.cub.2018.02.038 
Waller, D. (2000). Individual differences in spatial learning from computer-simulated environments. Journal of Experimental Psychology: Applied, 6(4), 307-321. https://doi.org/10.1037/1076-898X.6.4.307

Weisberg, S. M., \& Newcombe, N. S. (2016). How do (some) people make a cognitive map? Routes, places, and working memory. Journal of Experimental Psychology: Learning, Memory, and Cognition, 42(5), 768-785. http://dx.doi.org.proxy.library.ucsb.edu:2048/10.1037/xlm0000200

Wiener, J. M., Condappa, O. de, Harris, M. A., \& Wolbers, T. (2013). Maladaptive Bias for Extrahippocampal Navigation Strategies in Aging Humans. Journal of Neuroscience, 33(14), 6012-6017. https://doi.org/10.1523/JNEUROSCI.0717-12.2013

Wolbers, T., \& Hegarty, M. (2010). What determines our navigational abilities? Trends in Cognitive Sciences, 14(3), 138-146. https://doi.org/10.1016/j.tics.2010.01.001

Zhong, J. Y., \& Moffat, S. D. (2016). Age-Related Differences in Associative Learning of Landmarks and Heading Directions in a Virtual Navigation Task. Frontiers in Aging Neuroscience, 8. https://doi.org/10.3389/fnagi.2016.00122 


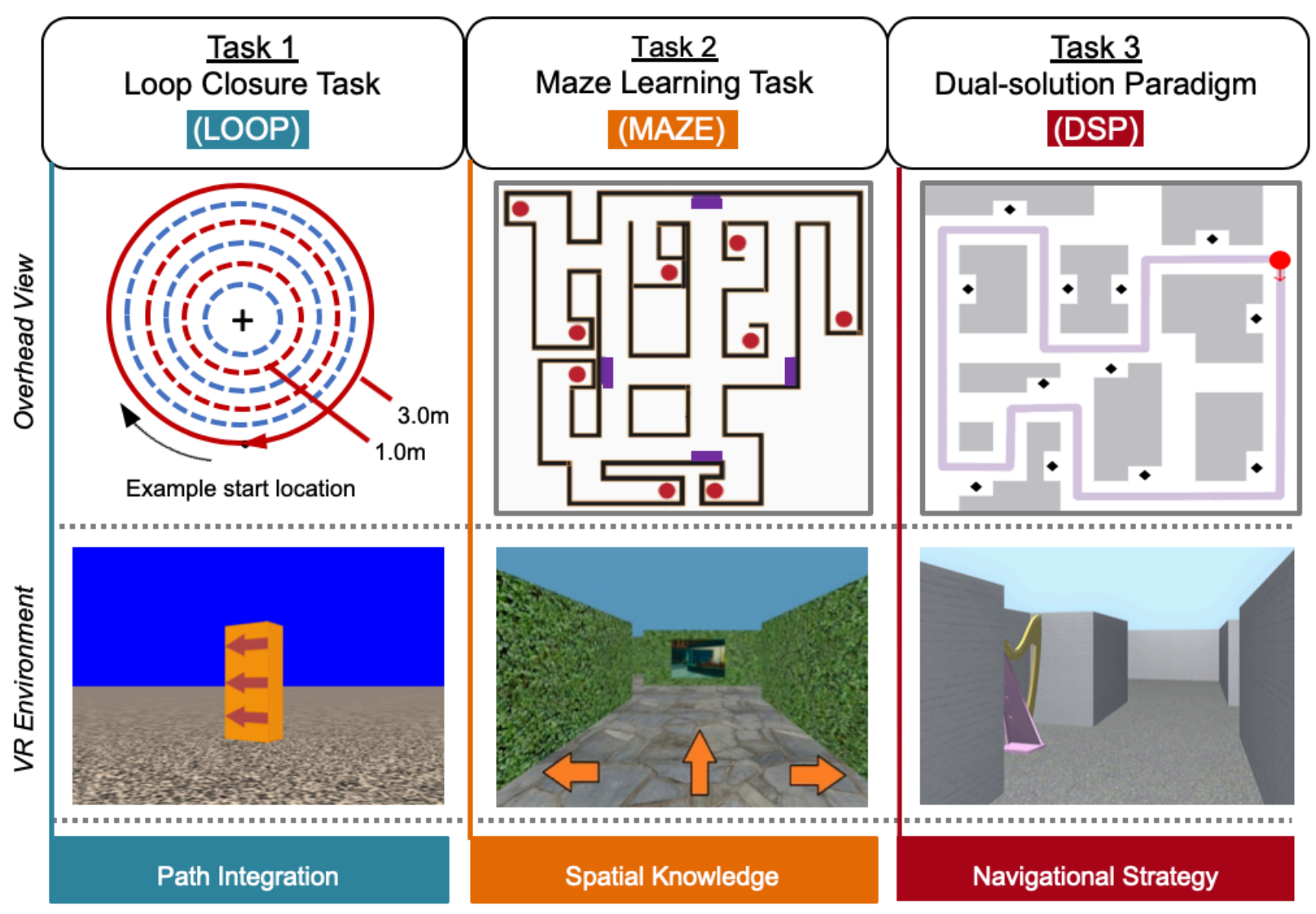

Figure 1. Three virtual reality tasks probe unique aspects of navigation ability. Task 1, the Loop Closure Task (LOOP), is a probe of path integration. Participants walk in a circle while tracking their start location. They indicate when they think they have returned to the start. Task 2, the Maze Learning Task (MAZE), tests spatial knowledge acquisition. Participants begin by freely exploring a novel maze environment and then during the test phase are asked to navigate to target locations without feedback. Task 3, the Dual-solution Paradigm (DSP), probes navigational strategy. Participants are first guided on a fixed route during the learning phase. During the test phase they are free to generate a novel shortcut to reach a target location or proceed along the learned path. 


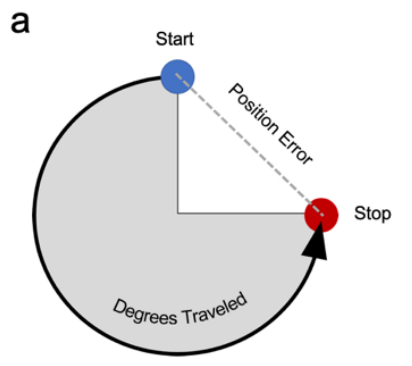

b

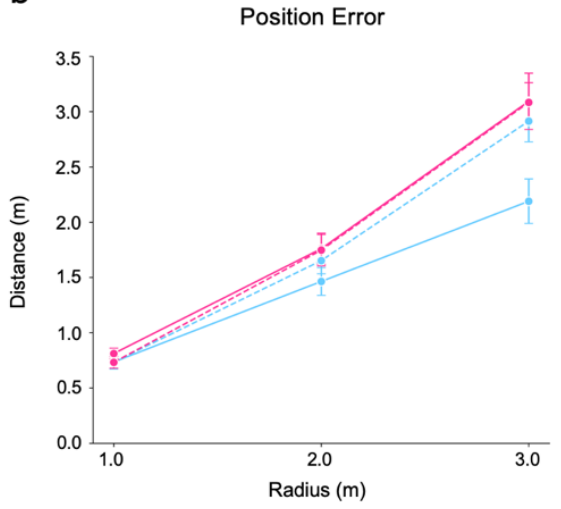

d

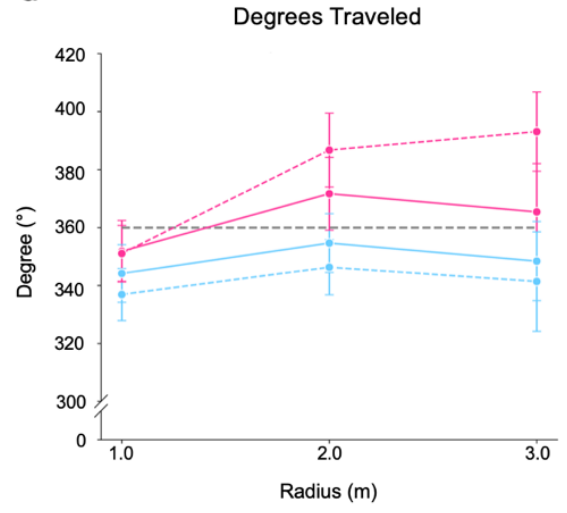

C

Position Error Variability

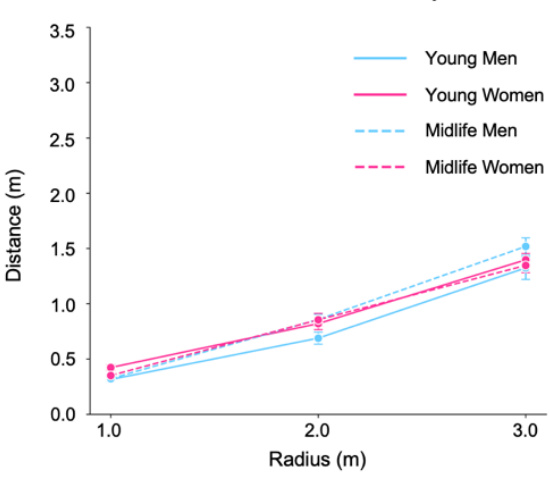

e

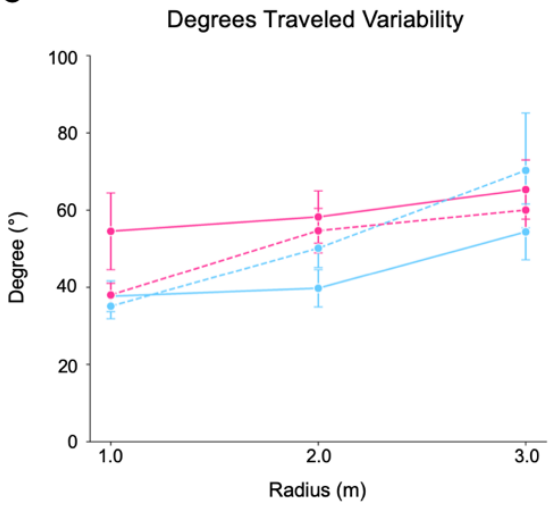

Figure 2. Minimal differences in path integration across sex or age. A. The blue dot indicates an example starting location along a circular loop and the red dot indicates an example stopping location in the LOOP task. Position error (represented by the grey dashed line between the blue and red dots) indicates the distance, in meters, between the participants' estimation of the starting position and the true starting position. Degrees traveled (represented by the black line on the circumference of the circle) indicates the total distance, in degrees, traversed by the participant. The arrow indicates the walking direction, which alternated between clockwise and counterclockwise. B. Position error and C. its variability increased as the radius size increased, but did not differ between young and midlife adults, nor between men and women. D. Degrees traveled as a measure of path integration differed by sex $(p=.018)$ with women tending to overshoot their estimated position along the circle and men undershooting. Degrees traveled did not differ between young and midlife adults. Grey dotted line at $360^{\circ}$ represents the ideal degrees participants should travel. E. Variability in degrees traveled did not differ between young and midlife adults, nor between men and women. (Young men: $n=27$; Young women: $n=25$; Midlife men: $n=23$; Midlife women: $n=33$ ). 

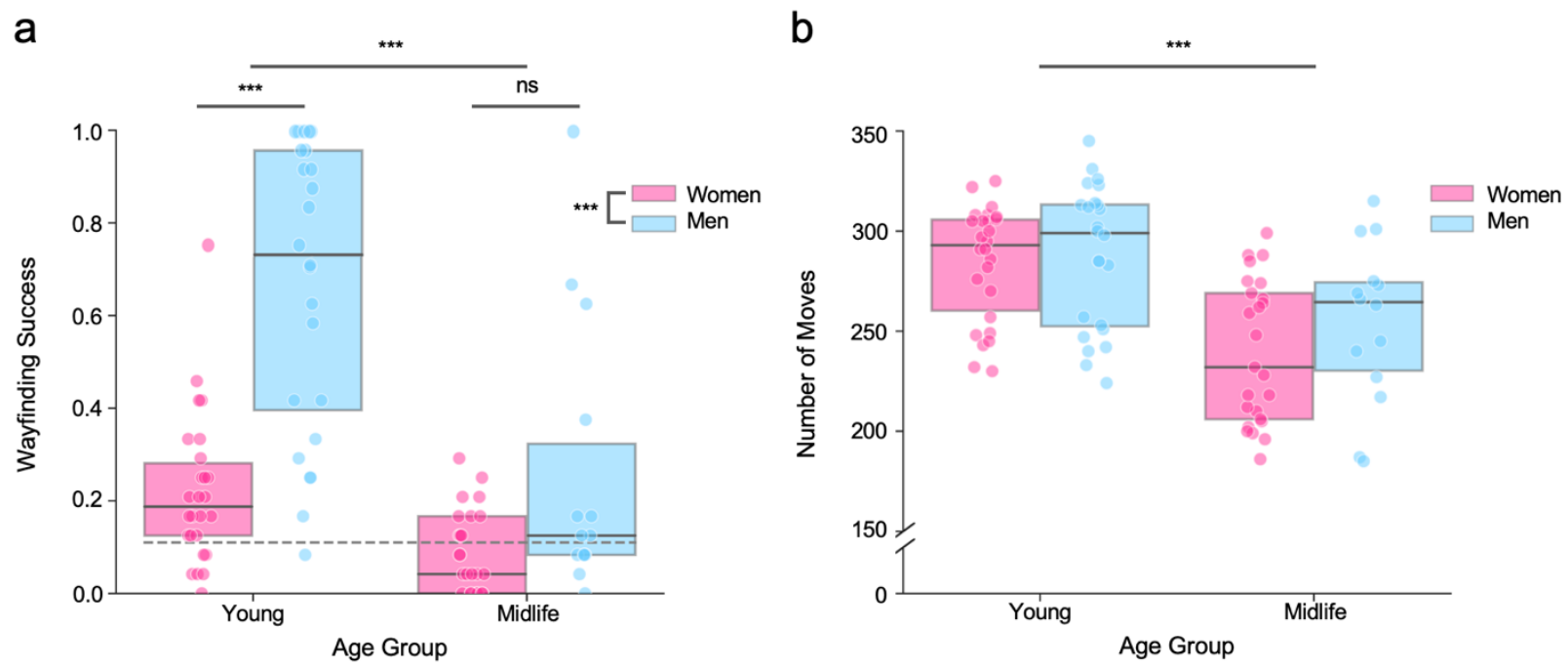

Figure 3. Spatial knowledge acquisition differs by age and sex. A. Wayfinding success in the MAZE task was used as a measure of spatial knowledge. After controlling for the number of moves made, young adults were more correct than midlife adults, and males were more correct overall than females. A sex difference that was robust in young adults did not persist with age. Grey dotted line at $11.11 \%$ represents chance level. B. Boxplots depict sex and age-related differences in number of moves during exploration in the MAZE task, indicating how much they explored the maze. Colored dots represent individual datapoints. Young adults made more moves than midlife adults, but there were no sex differences. (Young men: $n=24$; Young women: $n=$ 26; Midlife men: $n=14$; Midlife women: $n=25$ ). 
a

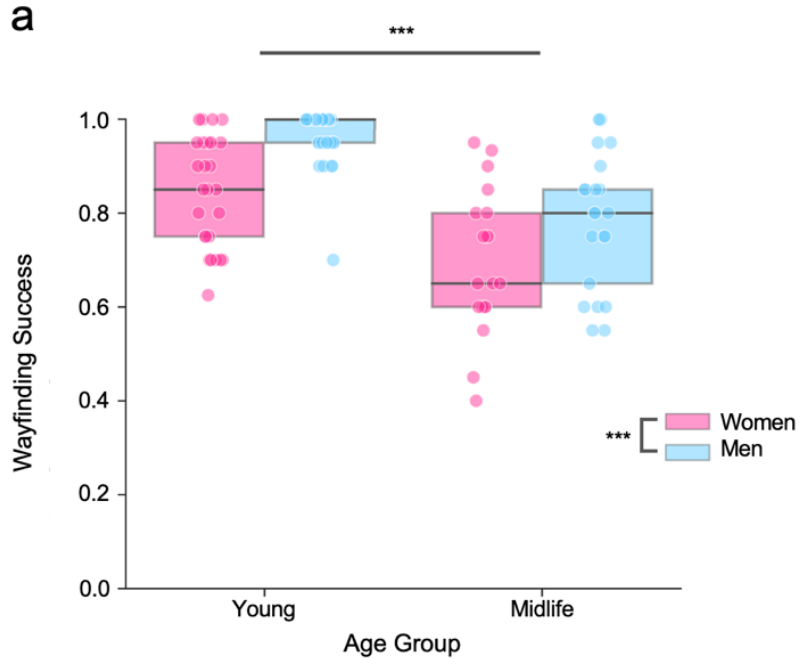

b

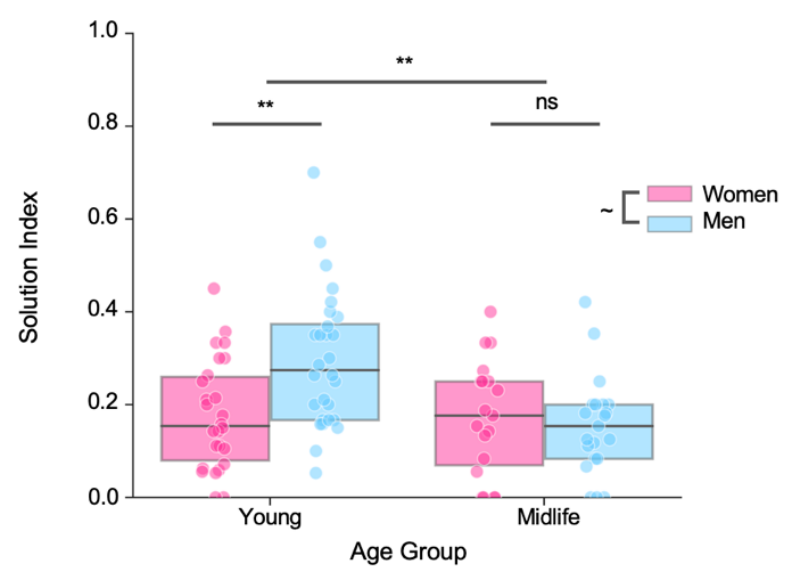

Figure 4. Navigational strategy differs by age and sex. A. Boxplots depict the proportion of successful trials in the DSP, indicated as wayfinding success, by age and sex. Colored dots represent individual datapoints. Midlife adults had fewer successful trials navigating to a target location relative to young adults. There was a main effect of sex, but no sex differences within young or midlife adults. B. The tendency to take shortcuts in the DSP, quantified here as the Solution Index (number of strict shortcuts/number of successful trials), was greater for young adults relative to midlife adults. Men overall took only marginally more shortcuts than women. Sex differences observed in young adults did not persist with age. (Young men: $n=28$; Young women: $n=26$; Midlife men: $n=21$; Midlife women: $n=19$ ). 


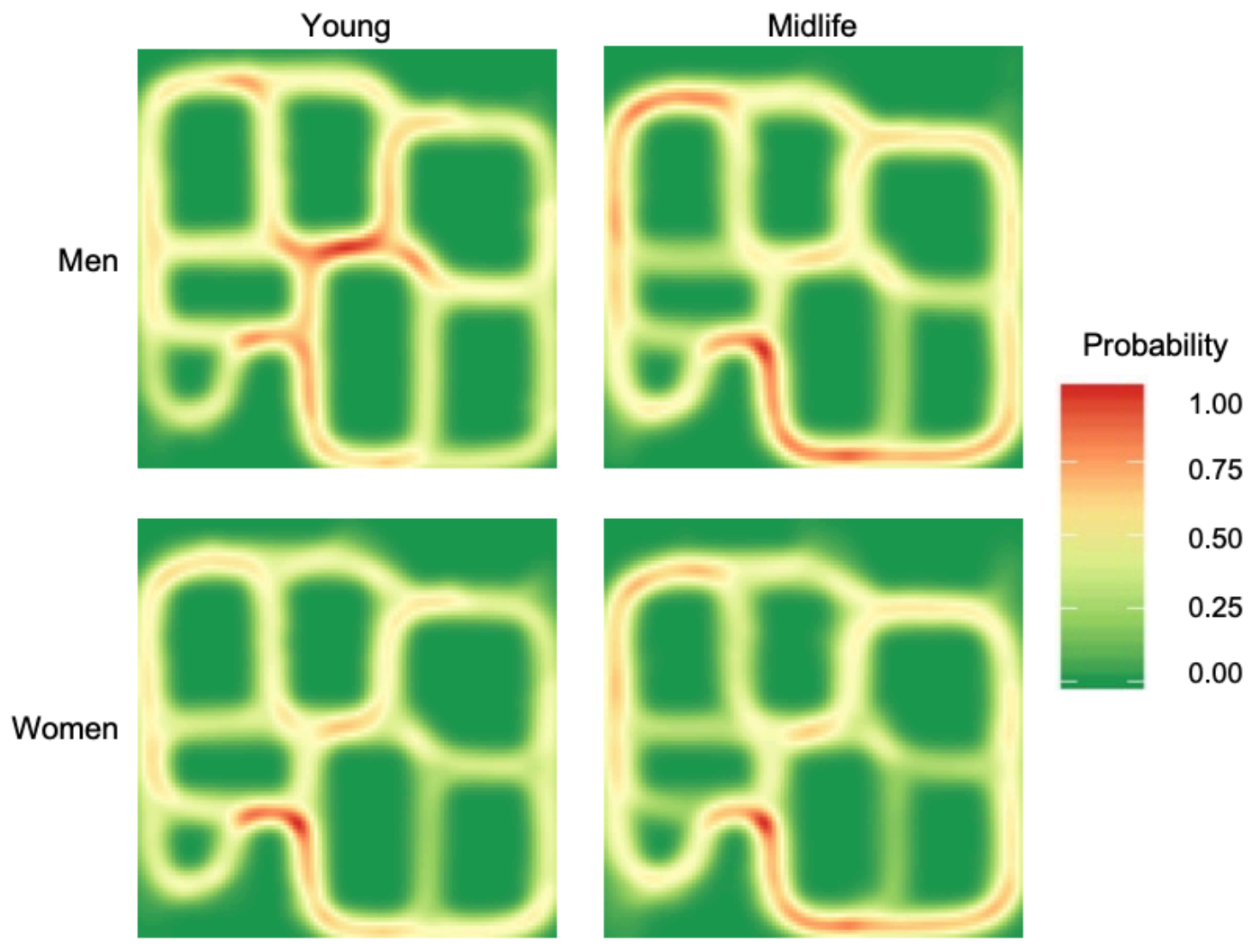

Figure 5. Heatmaps illustrate participants' tendency to take shortcuts. Young men $(\mathrm{n}=28)$ tend to take more shortcuts (as indicated by paths taken through the center of the maze), while young women $(\mathrm{n}=26)$, midlife men $(\mathrm{n}=21)$, and midlife women $(\mathrm{n}=19)$ tend to favor a learned route along the periphery. 